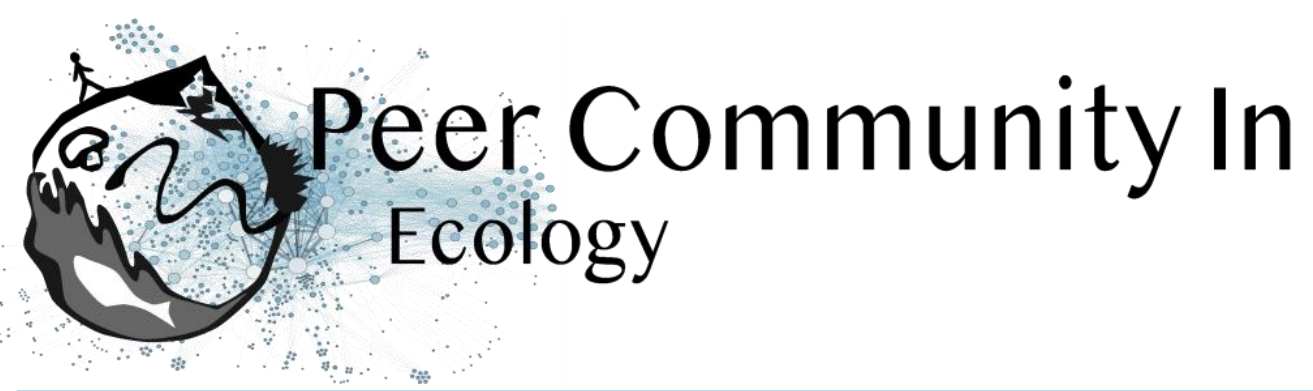

\title{
Tell us how you can be, and we'll make you better: exploiting genetic variability in personality traits to improve top-down control of agricultural pests
}

Marta Montserrat based on reviews by Joshua Patrick Byrne, François Dumont, Ana Pimenta Goncalves Pereira and Bart A Pannebakker

\section{Open Access}

\section{A recommendation of:}

Silène Lartigue, Myriam Yalaoui, Jean Belliard, Claire Caravel, Louise Jeandroz, Géraldine Groussier, Vincent Calcagno, Philippe Louâpre, François-Xavier DechaumeMoncharmont, Thibaut Malausa and Jérôme Moreau. Consistent variations in personality traits and their potential for genetic improvement of biocontrol agents:

Published: 14 January 2021

Copyright: This work is licensed under the Creative Commons Attribution-NoDerivatives 4.0 International License. To view a copy of this license, visit http://creativecommons.org/licen ses/by-nd/4.0/
Trichogramma evanescens as a case study (2020), bioRxiv, 2020.08.21.257881, ver. 4 peer-reviewed and recommended by Peer Community in Ecology. 10.1101/2020.08.21.257881

Submitted: 24 August 2020, Recommended: 13 January 2021

\section{Cite this recommendation as:}

Marta Montserrat (2021) Tell us how you can be, and we'll make you better: exploiting genetic variability in personality traits to improve top-down control of agricultural pests. Peer Community in Ecology, 100069. 10.24072/pci.ecology.100069

Agriculture in the XXI century faces the huge challenge of having to provide food to a rapidly growing human population, which is expected to reach 10.9 billion in 2100 (UUNN 2019), by means of practices and methods that guarantee crop sustainability, human health safety, and respect to the environment (UUNN 2015). Such regulation by the United Nations ultimately entails that agricultural scientists are urged to design strategies and methods that effectively minimize the use of harmful chemical products to control pest populations and to improve soil quality.

One of the most, if not the most, sustainable, safe, and environmentally friendly approach to apply against pests is Biological Pest Control (BPC, hereafter), that is, the use of natural enemies to control the populations of pest organisms. The concept of BPC is by no means new: long back to the 300 AC, Chinese farmers built bamboo bridges between citrus trees to facilitate the foraging of the ant species Oecophylla smaragdina to control lepidopteran citrus pests (Konishi and Ito, 1973); It is also nice to use this recommendation letter to recall and quote the words written in 1752 by the famous Swedish taxonomist, botanist and zoologist, Carl Linnaeus: "Every insect has its predator 
which follows and destroys it. Such predatory insects should be caught and used for disinfecting crop-plants" (Hörstadius (1974) apud Linnaeus 1752).

Acknowledging the many cases of successes from BPC along our recent history, it is also true that application of BPC strategies during the XX century suffered from wrong-doings, mainly when the introduced biological control agent (BCA, hereafter) was of exotic origin and with a generalist diet-breath; in some cases the release of exotic species resulted on global extinction, reduction in the range of distribution, reduction in the population abundance, and partial displacement, of native and functionally similar species, and interbreeding with them (reviewed in van Lenteren et al. 2006). One of the most famous cases is that of Harmonia axyridis, a coccinellid predator of Asian origin that caused important environmental damage in North America (reviewed in Koch \& Galvan, 2008).

Fortunately, after the implementation of the Nagoya protocol (CBD, 2011) importation of exotic species for BPC use was severely restricted and controlled, worldwide. Consequently, companies and agricultural scientist were driven to reinforce their focus and interest on the exploitation of native natural enemies, via the mass-rearing and release of native candidates (augmentative BPC), the conservation of landscapes near the crops to provide resources for natural enemies (i.e. conservation biological pest control), or via the exploitation of the genetic variability of BCAs, to create strains performing better at regulating pest populations under specific biotic or abiotic negative circumstances. Some of these cases are cited in Lartigue et al. (2020). The genetic improvement of BCAs is a strategy still in its infancy, but there is no doubt that the interest for it has significantly increased over the last 5 years (Lommen et al 2017, Bielza 2020, Leung et al 2020).

In my humble opinion, what makes the paper of Lartigue et al. (2020) a remarkable contribution to the field of genetic breeding of BCAs is that it opens a new window of opportunities to the field, by exploring the possibilities for artificial selection of behavioral traits (Réale et al. 2007) to "create" strains of natural enemies displaying behavioral syndromes (Sih et al. 2004) that makes them better at regulating pest populations. The behavioral approach for breeding BCAs can then be extended by crossing it with known abiotic and/or biotic hostile environments (e.g. warm and drought environments, presence of predators/competitors to the BCA, respectively) and engineer strains more prompt to display particular behavioral syndromes to help them to overcome the overall hostility of specific environments. I strongly believe that the approach proposed in Lartigue et al. (2020) will influence the future management of agricultural systems, where strategies including the genetic breeding of BCAs' behavior will contribute to create better guards and protectors of our crops.

\section{References}

Bielza, P., Balanza, V., Cifuentes, D. and Mendoza, J. E. (2020). Challenges facing arthropod biological control: Identifying traits for genetic improvement of predators in protected crops. Pest Manag Sci.

doi: https://doi.org/10.1002/ps.5857

CBD - Convention on Biological Diversity, 2011. The Nagoya Protocol on Access and Benefit-sharing, https://www.cbd.int/abs/doc/protocol/nagoya-protocol-en.pdf

Hörstadius, S. (1974). Linnaeus, animals and man. Biological Journal of the Linnaean Society, 6, 269-275.

doi: https://doi.org/10.1111/j.1095-8312.1974.tb00725.x

Koch, R.L. and Galvan, T.L. (2008). Bad side of a good beetle: the North American experience with Harmonia axyridis. BioControl 53, 23-35. doi: https://doi.org/10.1007/978-1-4020-6939-0 3

Konishi, M. and Ito, Y. (1973). Early entomology in East Asia. In: Smith, R.F., Mittler, T.E., Smith, C.N. (Eds.), History of Entomology, Annual Reviews Inc., Palo Alto, California, pp. 1-20.

Lartigue, S., Yalaoui, M., Belliard, J., Caravel, C., Jeandroz, L., Groussier, G., Calcagno, V., Louâpre, P., Dechaume-Moncharmont, F.-X., Malausa, T. and Moreau, J. (2020). Consistent variations in personality traits and their potential for genetic improvement of biocontrol agents: Trichogramma evanescens as a case study. bioRxiv, 2020.08.21.257881, ver. 4 peer-reviewed and recommended by PCI Ecology.

doi: https://doi.org/10.1101/2020.08.21.257881

Leung et al. (2020). Next-generation biological control: the need for integrating genetics and genomics. 
Biological Reviews, 95(6), 1838-1854. doi: https://doi.org/10.1111/brv.12641

Lommen, S. T. E., de Jong, P. W. and Pannebakker, B. A. (2017). It is time to bridge the gap between exploring and exploiting: prospects for utilizing intraspecific genetic variation to optimize arthropods for augmentative pest control - a review. Entomologia Experimentalis et Applicata, 162: 108-123.

doi: https://doi.org/10.1111/eea.12510

Réale, D., Reader, S. M., Sol, D., McDougall, P. T. and Dingemanse, N. J. (2007). Integrating animal temperament within ecology and evolution. Biological Reviews, 82: 291-318.

doi: https://doi.org/10.1111/i.1469-185X.2007.00010.x

Sih, A., Bell, A. and Johnson, J. C. (2004). Behavioral syndromes: an ecological and evolutionary overview. Trends in Ecology and Evolution, 19(7), 372-378. doi: https://doi.org/10.1016/j.tree.2004.04.009 UUNN. 2015. Transforming our world: the 2030 Agenda for Sustainable Development. report of the Open Working Group of the General Assembly on Sustainable Development Goals (A/68/970 and Corr.1; see also A/68/970/Add.1-3).

UUNN. 2019. World population prospects 2019. United Nations, Department of Economic and Social Affairs, Population Division: Highlights. ST/ESA/SER.A/423.

van Lenteren, J. C., Bale, J., Bigler, F., Hokkanen, H. M. T. and Loomans A. J. M. (2006). Assessing risks of releasing exotic biological control agents of arthropod pests. Annual Review of Entomology, 51: 609-634. doi: https://doi.org/10.1146/annurev.ento.51.110104.151129

\section{Revision round \#1}

2020-10-29

In the preprint presented by Lartigue et al. the authors introduce a novel perspective for the genetic improvement of Biological Control Agents (BCAs) based on behavioral traits that relate to the performance of individuals as BCAs, and that define the personality of individuals. The authors propose the genetic improvement of personality traits to be used as tool to create highly performant BCAs.

The idea is novel and the manuscript has potential to contribute significantly to agricultural sciences and to the development of new strategies to improve pest control with methodologies that are respectful with the environment and the human health.

Yet, in my opinion, the potential success for using behavioral traits to breed natural enemies is likely to be severely hampered by the extremely low heritability values $(<0.1)$ found in most of the behavioral traits and the two personality scores. This is a severe drawback that the authors need to address, not only by acknowledging the limitations of their strategy (as they do in the last paragraph of the discussion) but by offering real solutions, or convincing ways, to overcome this drawback, if only to convince the reader that introducing the genetic improvement of personalities in the biological pest control programs is realistic and feasible.

\section{Additional requirements of the managing board:}

As indicated in the 'How does it work?' section and in the code of conduct, please make sure that: -Data are available to readers, either in the text or through an open data repository such as Zenodo (free), Dryad or some other institutional repository. Data must be reusable, thus metadata or accompanying text must carefully describe the data.

-Details on quantitative analyses (e.g., data treatment and statistical scripts in R, bioinformatic pipeline scripts, etc.) and details concerning simulations (scripts, codes) are available to readers in the text, as appendices, or through an open data repository, such as Zenodo, Dryad or some other institutional repository. The scripts or codes must be carefully described so that they can be reused.

-Details on experimental procedures are available to readers in the text or as appendices.

-Authors have no financial conflict of interest relating to the article. The article must contain a "Conflict of interest disclosure" paragraph before the reference section containing this sentence: "The authors of this 


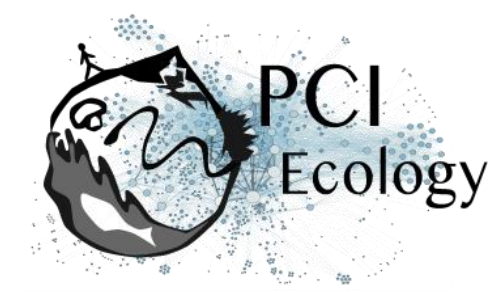

preprint declare that they have no financial conflict of interest with the content of this article." If appropriate, this disclosure may be completed by a sentence indicating that some of the authors are $\mathrm{PCl}$ recommenders: "XXX is one of the $\mathrm{PCI} X X X$ recommenders."

Preprint DOI: 10.1101/2020.08.21.257881

\section{Reviewed by Bart A Pannebakker, 2020-10-06 10:57}

Dear Martha,

I have read the paper "Consistent variations in personality traits and their potential for genetic improvement in the biocontrol agent Trichogramma evanescens" by Silène Lartigue et al. In their paper, the authors explore the variation in personality traits in minute Trichogramma wasps, for use in biological control. Animal personality is a relatively new field, and the authors are among the first to link this to the efficiency and potential for genetic improvement of biocontrol agents (BCA). Furthermore, the authors describe a highthroughput phenotyping system that allows the determination of the personality of the tiny wasps. Finally, they correlate the personality traits to more traditional used measures of biological control agent quality. All in all, I find this a very interesting manuscript that describes a new angle in the maintenance and improvement of quality of BCAs. Furthermore, the methodology is sound and solid and the paper is well written. I only have a few minor points which could help to further improve this paper:

I. 76: The reference should be = Wajnberg, Bernstein \& van Alphen, 2008

I. 108 \& I. 204: I am missing a good description of the personality traits that were investigated (boldness, activity and exploration). There is a reference to Reale et al 2007, but it would help the reader to provide a bit more definitions in the text.

I 184: Can you indicate why an LCD screen was used? It becomes clear in the text below, but it would help to guide the reader to explain that it was used to provide the surroundings in which to investigate the personality.

I.234-239: Can you shortly indicate what the different areas represent in the caption, so the figure can be understood without consulting the text?

L467-4670: It might seem very obvious that bigger females are more active. Bigger females have more fat, so more energy to spend. They therefore can use more energy to explore the area and don't need to save it for reproduction (or actually have more energy left for exploration). Can you include a discussion of this explanation, or am I wrong here?

I.523-525: Indeed, a low heritability might make selection on these traits difficult. One option is to move to genomic selection, as indicated in the recent paper by Leung et al. 2020 Next-generation biological control: the need for integrating genetics and genomics. Biol Rev. doi:10.1111/brv.12641. Genomic selection is especially useful when traits display a lower heritability. While this is currently still under development, it might be useful to shortly refer to genomic selection and/or the paper by Leung et al. 2020 here.

\section{Reviewed by François Dumont, 2020-09-18 18:53}

The proposed article is interesting, well written, and detailed. The approach is very relevant to the emerging field of biological control agent selection. However, researchers who will be interested in this approach do not require expertise in animal personalities. I think the authors need to explain better the link between the measures taken and their interpretation in terms of personality. The manuscript is also limited to a few examples of parasitoids, while BCA's genetic improvement targets more diverse groups and situations. Therefore, the article could have a broader impact if it better integrated other organisms such as predatory 
mite and omnivorous bugs. Besides, it will be interesting to link to more conventional BCA improvement studies based on pesticide resistance. Links between animal personality and pesticide resistance have already been established. It would be interesting to discuss it further here. Finally, the study of animal personality is relevant to understanding the impact of pest insects. How could the methods proposed in the manuscript apply to these pests?

Download the review (PDF file)

Reviewed by Loshua Patrick Byrne, 2020-10-28 16:12

Common review report with Ana Pereira (ASAPbio PeerReview Challenge 22/09/2020)

Download the review (PDF file)

Reviewed by Ana Pimenta Goncalves Pereira, 2020-10-28 16:13

Common review report with Joshua Byrne (ASAPbio PeerReview Challenge 22/09/2020)

Author's reply:

Download author's reply (PDF file) 